\title{
Association of lipoprotein levels with sleep apnea: role of autonomic dysfunction
}

\author{
Pavel Siarnik ${ }^{1}$, Adela Penesova ${ }^{2}$, Zofia Radikova ${ }^{2}$, Alzbeta Hluchanova ${ }^{3}$, Oto Hanus ${ }^{4}$, \\ Katarina KLOBUCNiKova ${ }^{1}$, Peter TurCani ${ }^{1}$, Branislav Kollar ${ }^{1}$ \\ ${ }^{1} 1^{\text {st }}$ Department of Neurology, Faculty of Medicine, Comenius University, Bratislava, Slovakia; ${ }^{2}$ Institute of Clinical and \\ Translational Research, Biomedical Research Center, Slovak Academy of Sciences, Bratislava, Slovakia; ${ }^{3}$ Department of \\ Neurology, University Hospital Bratislava, Bratislava, Slovakia; ${ }^{4}$ Department of Neurology, Central Military Hospital, \\ Ruzomberok, Slovakia \\ E-mail:palo.siarnik@gmail.com
}

Objectives. Although multiple mechanisms, including autonomic dysfunction, seem to link sleep-disordered breathing (SDB) with dyslipidemia in animal studies, the data in clinical studies are limited. The aim of this study was to explore the association of lipoprotein levels with SDB measures in healthy habitual snorers. We supposed that autonomic dysfunction is the linking mechanism.

Methods. We enrolled 110 previously healthy subjects with complaints of habitual snoring. To assess SDB, polysomnography was performed. Blood samples for the analysis of total cholesterol (TC), high-density lipoprotein (HDL), low-density lipoprotein cholesterol (LDL), and triglycerides (TG) were obtained in a fasting condition after the polysomnography. Baroreflex sensitivity (BRS) was used to assess the autonomic dysfunction.

Results. In stepwise multiple linear regression analysis, minimal nocturnal blood oxygen saturation (beta $=-0.240, \mathrm{p}=0.020$ ) and neck circumference (beta $=0.224, \mathrm{p}=0.03$ ) were the only significant contributors in model predicting TG. SDB measures were not identified as significant contributors in models predicting TC, LDL, and HDL. We failed to find any significant difference in BRS in SDB subjects when compared according to the presence or absence of hypercholesterolemia/ hypertriglyceridemia. In SDB subjects, the area under the curve in a receiver operating curve to predict hypercholesterolemia and hypertriglyceridemia by BRS was 0.468 (95\% CI: $0.328-0.608$ ) and 0.425 (95\% CI: 0.304-0.546), respectively.

Conclusions. Our results suggest that minimal nocturnal blood oxygen saturation is significant contributor in model predicting TG. No significant decrease in BRS was found in SDB subjects with hypercholesterolemia and hypertriglyceridemia. In SDB subjects, the role of autonomic dysfunction in the development of dyslipidemia remains controversial.

Key words: sleep-disordered breathing, polysomnography, total cholesterol, triglycerides, baroreflex sensitivity

The results from multiple animal studies show direct causal relationship between intermittent hypoxia and hyperlipidemia, with increases in lipids proportional to the severity of the hypoxia (Li et al. 2005; Li et al. 2007a). Mechanisms involved in dyslip- idemia induced by intermittent hypoxia may include up-regulation of lipid biosynthesis through up-regulation of sterol regulatory element binding protein 1c (SREBP-1c), a key hepatic transcription factor of lipid biosynthesis (Li et al. 2005; Li et al. 2007a, b;

Corresponding author: Pavel Siarnik MD. PhD., 1st Department of Neurology, Faculty of Medicine, Comenius University, Mickiewiczova 13, 81369, Bratislava, Slovakia; phone: +421903116499; fax: +421252967169; e-mail: palo.siarnik@gmail.com. 
Savransky et al. 2007). Impairment of lipoprotein clearance is other possible mechanism. Intermittent hypoxia inhibits triglyceride-rich lipoprotein clearance by activating angiopoietin-like protein-4 (Angptl-4) that is a potent inhibitor of lipoprotein lipase in the adipose tissue (Dallinga-Thie et al. 2010; Drager and Polotsky 2011). Intermittent hypoxia also activates the sympathetic nervous system that could potentially induce lipolysis (Jaworski et al. 2007; Jun and Polotsky 2007, 2009; Lafontan and Langin 2009). The evidence from the human studies linking SDB with dyslipidemia, although suggesting the role of SDB in development of dyslipidemia and favorable effect of positive airway pressure therapy, is still limited (Newman et al. 2001; Roche et al. 2009). Increased sympathetic drive resulting from recurrent nocturnal respiratory events is an important mechanism reported in the pathophysiology of SDB (Narkiewicz et al. 1998). Baroreflex system normally buffers increases in the blood pressure by inhibiting the sympathetic nervous system and it provides a negative feedback by aortic, carotid, and pulmonary baroreceptors (Chapleau 2012). Depressed baroreflex sensitivity (BRS) has been reported in SDB by multiple authors and it was supposed, that repeated sympathetic activation may change BRS in sleep apnea subjects and lead to chronic sympathetic overactivation (Siarnik et al. 2014; Cortelli et al. 2012). Other authors have supposed that sympathovagal imbalance in the form of increased sympathetic and decreased parasympathetic activity might contribute to the decreased BRS in the pre-obese and obese subjects (Indumathy et al. 2015). It is necessary to admit that clear evidence for causality between increased sympathetic activity and decreased BRS in sleep apnea is currently missing.

The aim of this study was to explore the association of lipoprotein levels with SDB measures in subjects with complaints of habitual snoring without previous diagnosis of SDB or other chronic disease. In population with newly diagnosed SDB, we aimed to compare BRS in subjects with hypercholesterolemia/hypertriglyceridemia compared to SDB subjects without hypercholesterolemia/hypertriglyceridemia. We are not aware of any similar study so far.

\section{Subjects and methods}

The study population was recruited from the patients examined in the sleep laboratory of the $1^{\text {st }}$ Department of Neurology, Comenius University and University Hospital, Bratislava, Slovakia due to complaints of habitual snoring. The subjects were excluded in a case of previous diagnosis of SDB, arterial hypertension, cardiovascular disease, diabetes mellitus or any other chronic diseases. Any current medication or smoking habit were additional exclusion criteria. Between November 2011 and May 2017, a total number of 110 subjects was recruited. Demographic data and clinical characteristics including age, gender, body-mass index (BMI), waist circumference, and neck circumference were recorded on admission. According to the values of BMI, subjects were classified as lean $(\mathrm{BMI}<25)$, overweight $(25 \leq \mathrm{BMI}<30)$ or obese $(\mathrm{BMI} \geq 30)$. The study was approved by the local ethics committee (Ethics committee of the University Hospital Bratislava) and all subjects signed an informed consent.

The sleep study was performed in standardized sleep laboratory settings using Alice 5 or Alice 6 devices (Philips-Respironics, Netherlands). Standardized criteria for scoring of the sleep parameters and respiratory events were used. Apnea was defined as the cessation or the reduction of airflow of $\geq 90 \%$ for $>10 \mathrm{~s}$ and hypopnea as a reduction in airflow of $\geq 50 \%$ for $10 \mathrm{~s}$ with oxygen desaturation of $\geq 3 \%$ or arousal. Apnea-hypopnea index (AHI; defined as number of apneas and hypopneas per hour of sleep), oxygen desaturation index (ODI; defined as number of desaturations $\geq 3 \%$ with duration $>10 \mathrm{~s}$ per hour of sleep) and blood oxygen saturation were recorded (Iber et al. 2007). SDB was defined as AHI $\geq 5$. Moderate-to-severe SDB (msSDB) was defined as $\mathrm{AHI} \geq 15$. Scorers were blinded to the baseline characteristics of the study population.

Blood tests were performed in the morning following polysomnography. The blood samples were obtained in fasting condition and processed in the local hospital laboratory. Parameters including total cholesterol (TC), high-density lipoprotein (HDL), low-density lipoprotein cholesterol (LDL), and triglycerides (TG) were set using an enzymatic colorimetric method (Cobas Mira Plus, Roche Diagnostics GmbH, Montclair, NJ, USA). Hypercholesterolemia was defined as TC>5 mmol/l and hypertriglyceridemia as TG $>1.7 \mathrm{mmol} / \mathrm{l}$.

A volume clamp method to capture beat-to-beat values of blood pressure and pulse rate in the finger artery was used for noninvasive measurement of BRS. A cuff of appropriate size was attached to the middle finger of the non-dominant hand and continuous blood pressure and pulse rate were recorded by using the Finometer device (Finapres Medical Systems BV, Amsterdam, Netherlands) as previously described (Sykora et al. 2009). Spontaneous BRS measurement was recorded for a period of $10 \mathrm{~min}$ and the value of 
BRS calculated by the Finometer device was used.

Categorical variables were expressed as numbers and proportion (\%), continuous variables as means ( \pm standard deviation) or median (interquartile range [IQR], minimal-maximal values) depending on normality of distribution. Pearson or Spearman correlation coefficients were used to determine the relationships between lipoprotein levels and the characteristics of the study population. Stepwise multiple linear regression was used to create the prediction model and identify the most important contributors to this model. A model including highest number of significant predictors was chosen. The dependent variable in each model was the levels of particular lipoprotein (TC, TG, LDL, HDL, separately). We included following baseline characteristics as independent variables in each model: age, gender, waist circumference, neck circumference, BMI, AHI, ODI, average, and minimal nocturnal saturation of blood with oxygen. Each model was assessed for the presence of multicollinearity of included variables. Vari- ance inflation factors (VIF) $\geq 5$ was indicative for multicollinearity.

For between-two-group comparison of particular variables, chi-squared test, Mann-Whitney test, and Student $t$ test were used. For comparison of multiple groups, the one-way analysis of variance (ANOVA) was used. All tests were two-sided and p-values $<0.05$ were considered as statistically significant. The receiver operating curve (ROC) was used to investigate the ability of BRS to predict hypercholesterolemia and hypertriglyceridemia in subjects with SDB. SPSS version 18 (SPSS Inc., Chicago, USA) was used for the statistical analyses.

\section{Results}

Our study population consisted of 19 females (17.3\%) and 91 males $(82.7 \%)$ with the history of habitual snoring. SDB was present in 87 patients (79.1\%) and msSDB in 54 subjects (49.1\%). Hypercholesterolemia was present in 64 patients (58.2\%) and

Table 1

Baseline characteristics of the study population.

\begin{tabular}{|c|c|c|c|c|}
\hline & Study population & SDB & No SDB & p-value \\
\hline $\mathrm{N}$ & 110 & $87(79.1 \%)$ & $23(20.9 \%)$ & \\
\hline Age (years) & $41.9 \pm 11.1$ & $43.0 \pm 11.2$ & $37.5 \pm 9.4$ & $0.033^{*}$ \\
\hline Females/males & $19 / 91(17.3 / 82.7 \%)$ & $12 / 75(13.8 / 86.2 \%)$ & $7 / 16(30.4 / 69.6 \%)$ & 0.060 \\
\hline Weight $(\mathrm{kg})$ & $92.9 \pm 18.6$ & $95.9 \pm 18.0$ & $81.7 \pm 16.6$ & $0.001^{\star *}$ \\
\hline Waist circumference $(\mathrm{cm})$ & $102.3 \pm 13.1$ & $104.7 \pm 12.5$ & $93.2 \pm 11.6$ & $<0.001^{\star * *}$ \\
\hline Neck circumference $(\mathrm{cm})$ & $40.4 \pm 4.2$ & $40.9 \pm 4.0$ & $38.1 \pm 4.1$ & $0.003^{* *}$ \\
\hline $\operatorname{BMI}\left(\mathrm{kg} / \mathrm{m}^{2}\right)$ & $29.7 \pm 5.5$ & $30.6 \pm 5.4$ & $26.2 \pm 4.5$ & $<0.001^{* * *}$ \\
\hline Total cholesterol $(\mathrm{mmol} / \mathrm{l})$ & $5.3 \pm 1.2$ & $5.4 \pm 1.2$ & $4.9 \pm 1.2$ & 0.089 \\
\hline Hypercholesterolemia (total cholesterol $>5 \mathrm{mmol} / \mathrm{l}$ ) & $64(58.2 \%)$ & $54(62.1 \%)$ & $10(43.5 \%)$ & 0.108 \\
\hline LDL cholesterol (mmol/l) & $3.8 \pm 1.0$ & $3.9 \pm 1.0$ & $3.4 \pm 1.1$ & $0.049^{*}$ \\
\hline HDL cholesterol (mmol/l) & $1.2 \pm 0.3$ & $1.2 \pm 0.3$ & $1.4 \pm 0.3$ & $0.019^{*}$ \\
\hline $\mathrm{TG}(\mathrm{mmol} / \mathrm{l})$ & $1.9 \pm 1.3$ & $2.0 \pm 1.4$ & $1.4 \pm 0.7$ & 0.055 \\
\hline Hypertriglyceridemia $(\mathrm{TG}>1.7 \mathrm{mmol} / \mathrm{l})$ & $49(44.5 \%)$ & $41(47.1 \%)$ & $8(34.8 \%)$ & 0.289 \\
\hline $\mathrm{AHI}(\mathrm{n} / \mathrm{h})$ & $14.3,23.9(0-101.3)$ & $20.7,30.8(5.1-101.3)$ & $1.3,3.7(0-4.6)$ & $<0.001^{* * *}$ \\
\hline ODI $(\mathrm{n} / \mathrm{h})$ & $13.4,24.1(0-119.9)$ & $20.5,25.7(5.2-119.9)$ & $1.7,3.1(0-6.9)$ & $<0.001^{\star * *}$ \\
\hline Average sat (\%) & $90.9 \pm 5.1$ & $90.0 \pm 5.4$ & $94.3 \pm 1.7$ & $<0.001^{\star * *}$ \\
\hline Minimal sat (\%) & $82.4 \pm 11.7$ & $80.3 \pm 12.2$ & $90.4 \pm 3.4$ & $<0.001^{* * *}$ \\
\hline Systolic BP (mmHg) & $125.7 \pm 11.6$ & $127.4 \pm 11.2$ & $119.3 \pm 11.2$ & $0.003^{\star *}$ \\
\hline Diastolic BP (mmHg) & $75.4 \pm 8.9$ & $76.3 \pm 8.9$ & $71.7 \pm 8.1$ & $0.026^{*}$ \\
\hline $\mathrm{BRS}(\mathrm{ms} / \mathrm{mmHg})$ & $8.2,7.1(2.7-47.7)$ & $7.3,6.2(2.7-34.2)$ & $13.0,11.0(4.8-47.7)$ & $<0.001^{\star * *}$ \\
\hline
\end{tabular}

Abbreviations: HDL - high-density lipoprotein; LDL - low-density lipoprotein; BMI - body-mass index; BP - blood pressure; AHI - apnea-hypopnea index; ODI - oxygen desaturation index; sat - saturation of blood with oxygen; BRS - baroreflex sensitivity; SDB - sleep-disordered breathing; TG - triglycerides. Categorical variables are expressed as numbers and proportions (\%); continuous variables as means \pm standard deviation or median, interquartile range, minimal and maximal values. ${ }^{*} \mathrm{p}<0.05,{ }^{* *} \mathrm{p}<0.01,{ }^{* *} \mathrm{p}<0.001$. 
hypertriglyceridemia in 49 patients (44.5\%). Hypercholesterolemia was present in 54 (62.1\%) and hypertriglyceridemia in 41 (47.1\%) patients with newly diagnosed SDB. Obesity was present in 50 subjects (45.5\%), 40 subjects (36.4\%) were overweight and 20 subjects $(18.2 \%)$ lean. Characteristics of the study population are shown in Table 1. Associations of lipoprotein levels with baseline characteristics of the study population are included in Table 2.

We observed weak to moderate positive correlation of TC levels with age and negative correlation of TC levels with average nocturnal saturation of blood with oxygen and minimal nocturnal saturation of blood with oxygen, see Table 2 . The model predicting TC in stepwise multiple linear regression analysis had $\mathrm{R}^{2}=0.083, \mathrm{p}=0.002$. Age (beta=0.289; $\mathrm{p}=0.002$ ) was the only significant contributor in this model. VIF of all variables assessed in this model were $<5$.

We observed weak to moderate positive correlation of LDL cholesterol levels with age and negative correlation with average nocturnal blood oxygen saturation, see Table 2. The model predicting LDL in stepwise multiple linear regression analysis had $\mathrm{R}^{2}=0.102, \mathrm{p}=0.001$. Age (beta=0.319, $\mathrm{p}=0.001$ ) was the only significant contributor in this model. VIF of all variables assessed in this model were $<5$.

We observed weak to moderate positive correlation of HDL cholesterol levels with age, average nocturnal blood oxygen saturation, minimal nocturnal blood oxygen saturation and inverse correlation with weight, waist circumference, neck circumference, BMI, AHI, ODI, systolic blood pressure, and diastolic blood pressure, see Table 2. As expected, men had significantly lower levels of HDL cholesterol than women $(1.1 \pm 0.3 \mathrm{mmol} / 1$ vs. $1.6 \pm 0.3 \mathrm{mmol} / \mathrm{l} ; \mathrm{p}<0.001)$. The model predicting $\mathrm{HDL}$ in stepwise multiple linear regression analysis had $\mathrm{R}^{2}=0.325, \mathrm{p}<0.001$. Neck circumference (beta $=-0.374, \mathrm{p}<0.001$ ) and male sex (beta $=-0.279, p=0.003$ ) were significant contributors in this model. VIF of all variables assessed in this model were $<5$.

We observed weak to moderate positive correlation of TG levels with weight, waist circumference, neck circumference, BMI, AHI, ODI, systolic blood pressure, and diastolic blood pressure as well as inverse correlation with average nocturnal blood oxygen saturation and minimal nocturnal blood oxygen saturation, see Table 2 . The model predicting TG in stepwise multiple linear regression analysis had $\mathrm{R}^{2}=0.161, \mathrm{p}<0.001$. Minimal nocturnal blood oxygen saturation (beta $=-0.240, \mathrm{p}=0.020$ ) and neck circumference (beta $=0.224, p=0.03$ ) were significant contributors in this model. VIF of all variables assessed in this model were $<5$.

The study population was divided into 4 groups according to the presence of SDB and hyperlipoproteinemia (hypercholesterolemia or hypertriglyceridemia), Figure 1 and Figure 2. No significant difference was found in the values of BRS in the subgroup with SDB and hypercholesterolemia compared to the

Table 2

Association of lipoprotein levels with baseline characteristics of the study population.

\begin{tabular}{|c|c|c|c|c|c|c|c|c|}
\hline & \multicolumn{2}{|c|}{ Total cholesterol } & \multicolumn{2}{|c|}{ LDL cholesterol } & \multicolumn{2}{|c|}{ HDL cholesterol } & \multicolumn{2}{|c|}{ Triglycerides } \\
\hline & $\mathbf{R}$ & p-value & $\mathbf{R}$ & p-value & $\mathbf{R}$ & p-value & $\mathbf{R}$ & p-value \\
\hline Age & 0.284 & $0.003^{\star \star}$ & 0.322 & $0.001^{* *}$ & 0.236 & $0.013^{\star *}$ & 0.057 & 0.554 \\
\hline Weight & -0.081 & 0.405 & -0.031 & 0.749 & -0.482 & $<0.001^{* * *}$ & 0.363 & $<0.001^{* * *}$ \\
\hline Waist circumference & 0.047 & 0.629 & 0.095 & 0.326 & -0.457 & $<0.001^{\star * *}$ & 0.496 & $<0.001^{\star * *}$ \\
\hline Neck circumference & -0.034 & 0.727 & -0.013 & 0.894 & -0.484 & $<0.001^{\star * *}$ & 0.431 & $<0.001^{\star * *}$ \\
\hline BMI & 0.117 & 0.223 & 0.141 & 0.142 & -0.363 & $<0.001^{\star * *}$ & 0.465 & $<0.001^{\star * *}$ \\
\hline AHI & 0.143 & 0.136 & 0.163 & 0.089 & -0.305 & $0.001^{\star *}$ & 0.280 & $0.003^{\star *}$ \\
\hline ODI & 0.136 & 0.157 & 0.167 & 0.081 & -0.337 & $<0.001^{\star * *}$ & 0.294 & $0.002^{\star *}$ \\
\hline Average sat & -0.235 & $0.013^{*}$ & -0.293 & $0.002^{\star *}$ & 0.237 & $0.013^{\star}$ & -0.381 & $<0.001^{\star * *}$ \\
\hline Minimal sat & -0.188 & $0.049^{*}$ & -0.160 & 0.096 & 0.255 & $0.007^{\star *}$ & -0.314 & $<0.001^{\star * *}$ \\
\hline Systolic BP & 0.075 & 0.438 & 0.046 & 0.631 & -0.298 & $0.002^{\star \star}$ & 0.247 & $0.009^{* *}$ \\
\hline Diastolic BP & 0.106 & 0.269 & 0.119 & 0.216 & -0.301 & $0.001^{\star \star}$ & 0.316 & $0.001^{\star *}$ \\
\hline
\end{tabular}

Abbreviations: HDL - high-density lipoprotein; LDL - low-density lipoprotein; BMI - body-mass index; BP - blood pressure; AHI apnea-hypopnea index; ODI - oxygen desaturation index; sat - saturation of blood with oxygen.

${ }^{*}$ p-value below $0.05 ;{ }^{* *}$ p-value below $0.01 ;{ }^{* *}$ p-value below 0.001 . 


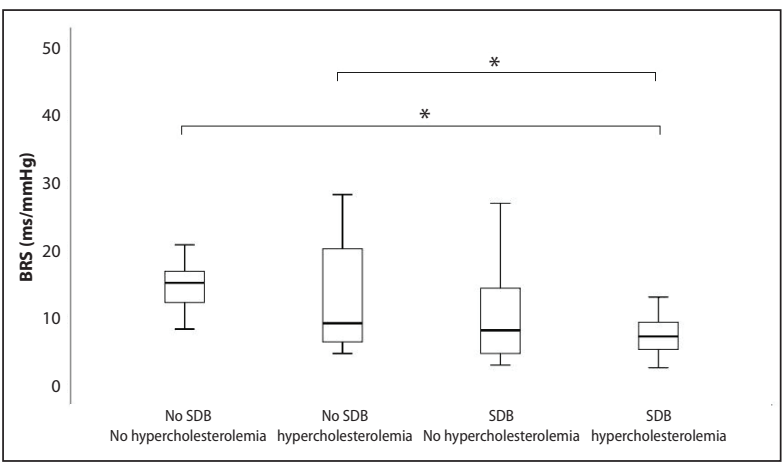

Figure 1. Baroreflex sensitivity (BRS) in groups divided according to the presence of sleep-disordered breathing (SDB) and hypercholesterolemia. Median, interquartile range, minimal and maximal values of BRS are presented in a graph. Median BRS (ms/mmHg): 15.2 in no SDB and no hypercholesterolemia; 9.3 in no SDB and hypercholesterolemia; 8.2 in SDB and no hypercholesterolemia; 7.3 in SDB and hypercholesterolemia. ${ }^{*} \mathrm{p}<0.05$.

subgroup with SDB and without hypercholesterolemia (median 7.3 vs. median $8.2 ; \mathrm{p}=0.463$ ). Similarly, no significant difference was found in the values of BRS in the subgroup with SDB and hypertriglyceridemia compared to the subgroup with SDB and without hypertriglyceridemia (median 6.5 vs. median 8.2; $\mathrm{p}=0.766$ ).

In subjects with $\mathrm{SDB}$, the area under the curve (AUC) in a ROC to predict hypercholesterolemia and hypertriglyceridemia by BRS was 0.468 (95\% CI: $0.328-0.608)$ and 0.425 (95\% CI: 0.304-0.546), respectively. Similarly, in a population with $\mathrm{msSDB}$, the AUC to predict hypercholesterolemia and hypertriglyceridemia by BRS was 0.507 (95\% CI: $0.322-$ 0.691 ) and 0.427 (95\% CI: 0.272-0.583), respectively.

\section{Discussion}

The results of our study suggest high prevalence of dyslipidemia in patients with newly diagnosed SDB who had no previous diagnosis of any other chronic disease. Hypercholesterolemia was present in $62.1 \%$ and hypertriglyceridemia in $47.1 \%$ of patients with newly diagnosed SDB. Our results suggest that minimal nocturnal blood oxygen saturation is significant contributor in model predicting TG. SDB measures were not identified as significant contributors in models predicting TC, LDL and HDL. BRS did not differ in SDB subjects with hypercholesterolemia/hypertriglyceridemia when compared to SDB subjects without hypercholesterolemia/hypertriglyceridemia. Similarly, the values of AUC in a ROC

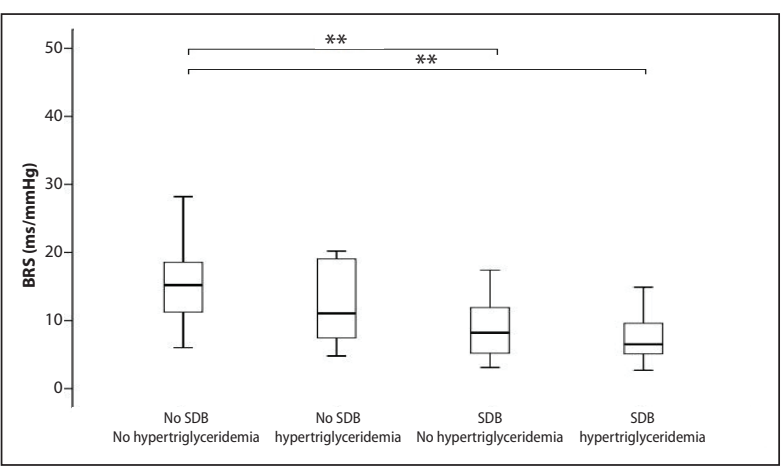

Figure 2. Baroreflex sensitivity (BRS) in groups divided according to the presence of sleep-disordered breathing (SDB) and hypertriglyceridemia. Median, interquartile range, minimal and maximal values of BRS are presented in a graph. Median BRS (ms/mmHg): 15.2 in no SDB and no hypertriglyceridemia; 11.1 in no SDB and hypertriglyceridemia; 8.2 in SDB and no hypertriglyceridemia; 6.5 in SDB and hypertriglyceridemia. ${ }^{* *} \mathrm{p}<0.01$.

suggested no ability of BRS to predict hypercholesterolemia/hypertriglyceridemia in subjects with SDB.

Hyperlipidemia is a well-known risk factor for the development of vascular diseases and it could count to the important mechanisms linking SDB with adverse cardiovascular outcomes (Oravec et al. 2011; Zhang et al. 2012; Ginter and Simko 2016). The evidence from the human studies linking SDB with dyslipidemia is limited and available studies are mostly cross-sectional and non-randomized (Drager et al. 2010). The largest meta-analysis so far includes sixty-four studies, 7971 SDB subjects and 10145 controls. Results of the meta-analysis suggested that patients with SDB have increased dyslipidemia, as characterized by high TC, LDL, TG, and low HDL. Meta-regression for age, BMI, and AHI showed that AHI had significant effect for LDL and TG. In summary, it appears that there is some evidence indicating higher degree of dyslipidemia in patients with SDB that may correlate with the severity of disease. However, this relationship is probably confounded by multiple factors including weight, BMI, body fat, and comorbidities (Nadeem et al. 2014). The results of the meta-analysis of 6 randomized controlled trials focusing on positive airway pressure (PAP) therapy suggest that application of PAP significantly reduced the TC level, but not the levels of TG, LDL, or HDL (Xu et al. 2014).

Our results are in concordance with the results of previous studies. In a study involving 275 adult patients with complaints of habitual snoring and without previous diagnosis of SDB, $85 \%$ were diag- 
nosed with SDB, as defined by AHI > 5. Similarly, to our study, patients with severe cardiovascular disease, liver cirrhosis, end-stage renal disease, chronic obstructive pulmonary disease, hematological disease or cancer were excluded. The prevalence of hypercholesterolemia and hypertriglyceridemia was, similarly to our study, $61.1 \%$ and $55.3 \%$, respectively. Moreover, authors have found ODI to be an independent factor contributing to hypercholesterolemia and hypertriglyceridemia (Chou et al. 2010). Selection of subjects without previous diagnosis of SDB or other chronic disease in our study could help to eliminate confounding from other associated conditions or medications. We found that minimal nocturnal saturation of blood with oxygen and neck circumference were significant contributors in this model predicting TG levels. SDB measures were not identified as significant contributors in models predicting TC, LDL and HDL.

Causal relationship of SDB and dyslipidemia remains unclear. There is an increasing evidence from the animal studies that intermittent hypoxia, a key-player in a pathophysiology of SDB, is independently associated with dyslipidemia via generation of stearoyl-coenzyme A desaturase-1, production of reactive oxygen species, peroxidation of lipids, and sympathetic system dysfunction (Adedayo et al. 2014). The clinical evidence has to be elucidated. We hypothesize that autonomic dysfunction could be an important linking mechanism in our population. Increased sympathetic drive resulting from recurrent nocturnal respiratory events is an important mechanism reported in the pathophysiology of SDB (Narkiewicz et al. 1998). Depressed BRS has been reported in SDB by multiple authors (Ryan et al. 2007; Cortelli et al. 2012; Siarnik et al. 2014). Clear evidence for causality between increased sympathetic activity and decreased BRS in sleep apnea is currently missing. However, it has been supposed that repeated sympathetic activation may change BRS in sleep apnea subjects and lead to a chronic sympathetic overactivation (Cortelli et al. 2012). To the best of our knowledge this was the first study so far to search for differences in BRS in subjects with newly diagnosed SDB and hypercholesterolemia/ hypertriglyceridemia compared to SDB subjects without hypercholesterolemia/hypertriglyceridemia. Despite lower values of BRS in SDB populations with hypercholesterolemia/hypertriglyceridemia compared to the SDB populations without hypercholesterolemia/hypertriglyceridemia, these differences failed to reach the statistical significance. When simplified, populations with the presence of
SDB and/or hyperlipidemia reached lower values of BRS when compared to the populations without such disorders. Not all the differences reached the statistical significance (for details see Figure 1 and Figure 2). Similarly, the values of AUC in a ROC suggest no ability of BRS to predict hypercholesterolemia/hypertriglyceridemia in subjects with SDB. Non-significant difference in BRS values in SDB populations with hyperlipidemia in comparison to SDB populations without hyperlipidemia may have several explanations:

1. Lipolysis induced by sympathetic activation may not be one of the leading mechanisms linking SDB with dyslipidemia. Several other potentially more important mechanisms were described in the animal studies, including lipid peroxidation, up-regulation of lipid biosynthesis through up-regulation of SREBP-1c or impairment of lipoprotein clearance by activating Angptl-4, a potent inhibitor of lipoprotein lipase (Barcelo et al. 2000; Schulz et al. 2000; Li et al. 2005; Li et al. 2007a, b; Savransky et al. 2007; Dallinga-Thie et al. 2010; Drager and Polotsky 2011).

2. Relationship between hyperlipidemia and decreased BRS may be bidirectional. Hypercholesterolemia is a well-known risk factor for the development of atherosclerosis and atherosclerotic changes of the carotid arteries are associated with decreased BRS (Kwiterovich 2002; Nasr et al. 2005). Improvement of BRS has also been observed in the statin-treated subjects (Patterson et al. 2002; Celovska et al. 2016). Additionally, despite role of repeated sympathetic activation in change of BRS and following chronic sympathetic overactivation was supposed (Cortelli et al. 2012), this role still remains controversial. Decrease of BRS does not necessarily reflect sympathetic activation.

3. Although we tried to avoid confounders by enrollment of subjects without previous diagnosis of SDB or other chronic disease, the relationship between dyslipidemia and autonomic dysfunction may be confounded by multiple other factors including weight, fat mass, BMI or physical activity (La Rovere and Pinna 2014; Lira et al. 2014; Nadeem et al. 2014; Indumathy et al. 2015).

Cross-sectional nature belongs to the limitations of our study. Future longitudinal randomized clinical trials are needed to elucidate mechanisms linking SDB and dyslipidemia. Effect of PAP therapy on autonomic dysfunction and dyslipidemia should also be explored. 
Our results suggest that minimal nocturnal blood oxygen saturation is significant contributor in model predicting TG. SDB measures were not identified as significant contributors in models predicting TC, LDL and HDL. Decreased BRS, did not differ in SDB subjects with hypercholesterolemia/hypertriglyceridemia when compared to SDB subjects without hypercholesterolemia/hypertriglyceridemia, suggesting minor role of autonomic dysfunction in development of dyslipidemia in SDB subjects.

\section{Acknowledgements}

This research was supported by the Grant of the Framework Program for Research and Technological Development, Project: Completion of the Center of Excellence in Sudden Cerebrovascular Accidents, Faculty of Medicine, Comenius University in Bratislava (ITMS: 26240120023), co-financed by the European Regional Development Fund and by VEGA 2/0072/18 Grant.

\section{References}

Adedayo AM, Olafiranye O, Smith D, Hill A, Zizi F, Brown C, Jean-Louis G. Obstructive sleep apnea and dyslipidemia: evidence and underlying mechanism. Sleep Breath 18, 13-18, 2014.

Barcelo A, Miralles C, Barbe F, Vila M, Pons S, Agusti AG. Abnormal lipid peroxidation in patients with sleep apnoea. Eur Respir J 16, 644-647, 2000.

Celovska D, Kruzliak P, Rodrigo L, Gonsorcik J, Sabaka P, Gaspar P, Delev D, Petrovic D, Dukat A, Gaspar L. Effect of low dose Atorvastatin therapy on baroreflex sensitivity in hypertensives. High Blood Press Cardiovasc Prev 23, 133-140, 2016.

Cortelli P, Lombardi C, Montagna P, Parati G. Baroreflex modulation during sleep and in obstructive sleep apnea syndrome. Auton Neurosci 169, 7-11, 2012.

Dallinga-Thie GM, Franssen R, Mooij HL, Visser ME, Hassing HC, Peelman F, Kastelein JJ, Peterfy M, Nieuwdorp M. The metabolism of triglyceride-rich lipoproteins revisited: new players, new insight. Atherosclerosis 211, $1-8,2010$.

Drager LF, Jun J, Polotsky VY. Obstructive sleep apnea and dyslipidemia: implications for atherosclerosis. Curr Opin Endocrinol Diabetes Obes 17, 161-165, 2010.

Drager LF, Polotsky VY. Lipid metabolism: a new frontier in sleep apnea research. Am J Respir Crit Care Med 184, 288-290, 2011.

Ginter E, Simko V. New data on harmful effects of trans-fatty acids. Bratisl Lek Listy 117, 251-253, 2016.

Chapleau MW. Baroreceptor Reflexes. Sympatho-vagal imbalance in hypertension. In: Primer on the autonomic nervous system (Ed. D Robertson), pp. 161-165, Elsevier, San Diego, 2012.

Chou YT, Chuang LP, Li HY, Fu JY, Lin SW, Yang CT, Chen NH. Hyperlipidaemia in patients with sleep-related breathing disorders: prevalence \& risk factors. Ind J Med Res 131, 121, 2010.

Iber CAIS, Chesson A, Quan SF. The AASM Manual for the scoring of sleep and associated events: Rules, terminology and technical specification. American Academy of Sleep Medicine, Westchester, 2007.

Indumathy J, Pal GK, Pal P, Ananthanarayanan PH, Parija SC, Balachander J, Dutta TK. Decreased baroreflex sensitivity is linked to sympathovagal imbalance, body fat mass and altered cardiometabolic profile in pre-obesity and obesity. Metabolism 64, 1704-1714, 2015.

Jaworski K, Sarkadi-Nagy E, Duncan RE, Ahmadian M, Sul HS. Regulation of triglyceride metabolism. IV. Hormonal regulation of lipolysis in adipose tissue. Am J Physiol Gastrointest Liver Physiol 293, 1-4, 2007.

Jun J, Polotsky VY. Sleep disordered breathing and metabolic effects: evidence from animal models. Sleep Med Clin 2, 263-277, 2007.

Jun J, Polotsky VY. Metabolic consequences of sleep-disordered breathing. Ilar J 50, 289-306, 2009.

Kwiterovich PO, Jr. Lipoprotein heterogeneity: diagnostic and therapeutic implications. Am J Cardiol 90, 1-10, 2002.

La Rovere MT, Pinna GD. Beneficial effects of physical activity on baroreflex control in the elderly. Ann Noninvasive Electrocardiol 19, 303-310, 2014.

Lafontan M, Langin D. Lipolysis and lipid mobilization in human adipose tissue. Prog Lipid Res 48, 275-297, 2009.

Li J, Thorne LN, Punjabi NM, Sun CK, Schwartz AR, Smith PL, Marino RL, Rodriguez A, Hubbard WC, O’Donnell CP, Polotsky VY. Intermittent hypoxia induces hyperlipidemia in lean mice. Circ Res 97, 698-706, 2005.

Li J, Savransky V, Nanayakkara A, Smith PL, O’Donnell CP, Polotsky VY. Hyperlipidemia and lipid peroxidation are dependent on the severity of chronic intermittent hypoxia. J Appl Phisiol (1985) 102, 557-563, 2007a.

Li J, Nanayakkara A, Jun J, Savransky V, Polotsky VY. Effect of deficiency in SREBP cleavage-activating protein on lipid metabolism during intermittent hypoxia. Physiol Genomics 31, 273-280, 2007b. 
Lira FS, Rosa Neto JC, Antunes BM, Fernandes RA. The relationship between inflammation, dyslipidemia and physical exercise: from the epidemiological to molecular approach. Curr Diabetes Rev 10, 391-396, 2014.

Nadeem R, Singh M, Nida M, Waheed I, Khan A, Ahmed S, Naseem J, Champeau D. Effect of obstructive sleep apnea hypopnea syndrome on lipid profile: a meta-regression analysis. J Clin Sleep Med 10, 475-489, 2014.

Narkiewicz K, van de Borne PJH, Cooley RL, Dyken ME, Somers VK. Sympathetic activity in obese subjects with and without obstructive sleep apnea. Circulation 98, 772-776, 1998.

Nasr N, Pavy-Le Traon A, Larrue V. Baroreflex sensitivity is impaired in bilateral carotid atherosclerosis. Stroke 36, 1891-1895, 2005.

Newman AB, Nieto FJ, Guidry U, Lind BK, Redline S, Pickering TG, Quan SF. Relation of sleep-disordered breathing to cardiovascular disease risk factors: the Sleep Heart Health Study. Am J Epidemiol 154, 50-59, 2001.

Oravec S, Dukat A, Gavornik P, Caprnda M, Kucera M, Ocadlik I. Contribution of the atherogenic lipoprotein profile to the development of arterial hypertension. Bratisl Lek Listy 112, 4-7, 2011.

Patterson D, Dick JB, Struthers AD. Intensive statin treatment improves baroreflex sensitivity: another cardioprotective mechanism for statins? Heart 88, 415-416, 2002.

Roche F, Sforza E, Pichot V, Maudoux D, Garcin A, Celle S, Picard-Kossovsky M, Gaspoz JM, Barthelemy JC. Obstructive sleep apnoea/hypopnea influences high-density lipoprotein cholesterol in the elderly. Sleep Med 10, 882-886, 2009.

Ryan S, Ward S, Heneghan C, McNicholas WT. Predictors of decreased spontaneous baroreflex sensitivity in obstructive sleep apnea syndrome. Chest 131, 1100-1107, 2007.

Savransky V, Nanayakkara A, Li J, Bevans S, Smith PL, Rodriguez A, Polotsky VY. Chronic intermittent hypoxia induces atherosclerosis. Am J Respir Crit Care Med 175, 1290-1297, 2007.

Schulz R, Mahmoudi S, Hattar K, Sibelius U, Olschewski H, Mayer K, Seeger W, Grimminger F. Enhanced release of superoxide from polymorphonuclear neutrophils in obstructive sleep apnea. Impact of continuous positive airway pressure therapy. Am J Respir Crit Care Med 162, 566-570, 2000.

Siarnik P, Carnicka Z, Krizova L, Wagnerova H, Sutovsky S, Klobucnikova K, Kollar B, Turcani P, Sykora M. Predictors of impaired endothelial function in obstructive sleep apnea syndrome. Neuro Endocrinol Lett 35, 142-148, 2014.

Sykora M, Diedler J, Rupp A, Turcani P, Steiner T. Impaired baroreceptor reflex sensitivity in acute stroke is associated with insular involvement, but not with carotid atherosclerosis. Stroke 40, 737-742, 2009.

$\mathrm{Xu} \mathrm{H}$, Yi H, Guan J, Yin S. Effect of continuous positive airway pressure on lipid profile in patients with obstructive sleep apnea syndrome: a meta-analysis of randomized controlled trials. Atherosclerosis. 234, 446-453, 2014.

Zhang W, Sun Y, Li T, Zhang G, Wang Y, Sun H. The effect of obstructive sleep apnea-hypopnea syndrome on acute myocardial infarction. Bratisl Lek Listy 113, 565-568, 2012. 